\title{
Deconvolution Processing in Analytical STEM: Monochromator for EELS and Cs-Corrector for STEM-HAADF
}

\author{
Kazuo Ishizuka \\ HREM Research Inc, Matsukazedai, Saitama 355-0055, Japan
}

Improvement of energy-resolution and spatial-resolution has become possible by development of electron-gun monochromators and objective-lens Cs-correctors, respectively. However, their diffusion seems to be slow due to their high costs. On the other hand, an electron energy-loss spectrum (EELS) or a high-angle annular darkfield (HAADF) image of scanning transmission electron microscope (STEM) will be described by a convolution of an ideal spectrum or a scattering object with an instrument function. Therefore, a process of deconvolution will function as a monochromator for EELS or a Cs-corrector for STEM-HAADF. In this report, we will present some results obtained with indirect iterative deconvolution procedures based on the maximumentropy method (MEM) [1] and the Richardson-Lucy algorithm (RLA) [2].

An observed EELS intensity may be described within the first approximation as a sum of ideal EELS for each primary electron including the one suffered from energy loss. Thus, an observed EELS $\operatorname{Obs}(E)$ is written by a convolution between a low-loss spectrum $\operatorname{Low}(E)$ and an ideal EELS (Core-loss spectrum) $\operatorname{Core}(E)$ [3]:

$$
\operatorname{Obs}(E)=\int \operatorname{Low}(E-t) \operatorname{Core}(t) d t
$$

On the other hand, the STEM-HAADF signal is believed to be generated by electrons suffered from thermal diffuse scattering (TDS). Thus, using an absorption potential representing TDS electrons scattered into a HAADF detector, a HAADF image as a function of the probe position $\mathbf{r}_{p}$ will be described as follows [4]:

$$
i\left(\mathbf{r}_{p}\right)=\int p_{3}\left(\mathbf{r}-\mathbf{r}_{p}, z\right) o_{3}(\mathbf{r}, z) d \mathbf{r} d z
$$

where $p_{3}(\mathbf{r}, z)$ is a probe function propagating along the $\mathrm{z}$ direction, and $o_{3}(\mathbf{r}, z)$ a three-dimensional TDS HAADF absorption potential. When we can assume a single TDS event and no significant change of the probe shape within the sample, the observed HAADF image is described by the probe function $p(\mathbf{r})$ and the HAADF object function $o(\mathbf{r})$ :

$$
i\left(\mathbf{r}_{p}\right)=\int p\left(\mathbf{r}-\mathbf{r}_{p}\right) o(\mathbf{r}) d \mathbf{r} \equiv p\left(-\mathbf{r}_{p}\right) \otimes o\left(\mathbf{r}_{p}\right)
$$

Here, we may note that this equation is actually a cross-correlation, and it becomes a convolution integral only when the probe function is symmetric.

Since deconvolution tries to recover high-frequency information attenuated by an instrument function, the result of deconvolution becomes shaper than the experimental observation. Thus, deconvolution will usually amplify noise under the presence of noise. Contrary to this, both MEM and RLA deconvolution procedures will not amplify noise so much and can suppress an effect from un-physical high-frequency noise. For this purpose we will reduce un-physical noise by convoluting an observed data with a narrow smooth function, say a Gaussian, before performing deconvolution. Then, this artificially blurred data will be deconvoluted by an instrument function convoluted by the same smoothing function. This technique is especially useful for STEM-HAADF, since a signal-to-noise ratio of a HAADF image is rather low. Figure 1 shows a result of deconvolution of a STEM-HAADF image obtained by using JEOL 2010F. Here, we apply pre-convolution, and the deconvoluted image shows faint spots extend to $13 \mathrm{~nm}^{-1}$. Contrary to HAADF images the signal-tonoise ratio of an EELS is usually high, and thus pre-convolution will not affect the result of deconvolution [5]. However, Fig. 2 shows that pre-convolution can reduce electron dose to take an EELS by one order of magnitude without affecting the result of deconvolution.

We may note that deconvolution shown here will be useful even for the data obtained from a microscope equipped with a monochromator and/or Cs-corrector, since an energy width and a probe shape will never become a delta function. All the results shown in this report were obtained by using DeConvEELS [5] and 
DeConvHAADF [6], both of which are plug-ins for DigitalMicrograph [7].

\section{References}

[1] D.M. Colin, nature 298 (1982) 49.

[2] W.H. Richardson, J Opt Soc Am 62 (1972) 55; L.B. Lucy, Astrophysical Journal 79 (1974) 745.

[3] R.F. Egerton, Electron energy-loss spectroscopy in the electron microscope, Plenum Press, New York, 1986.

[4] K. Ishizuka, Ultramicroscopy 90 (2002) 71.

[5] K. Ishizuka, K. Kimoto, Y. Bando, Micros. Microanal. 9 (suppl. 2) (2003) CD832.

[6] K. Ishizuka, E. Abe, EMC 2004, I (2004) 117.

[7] Gatan Inc (www.gatan.com)

[8] The author greatly acknowledges Dr. E. Abe and Dr. P. Batson for providing the data to test the procedures presented here.
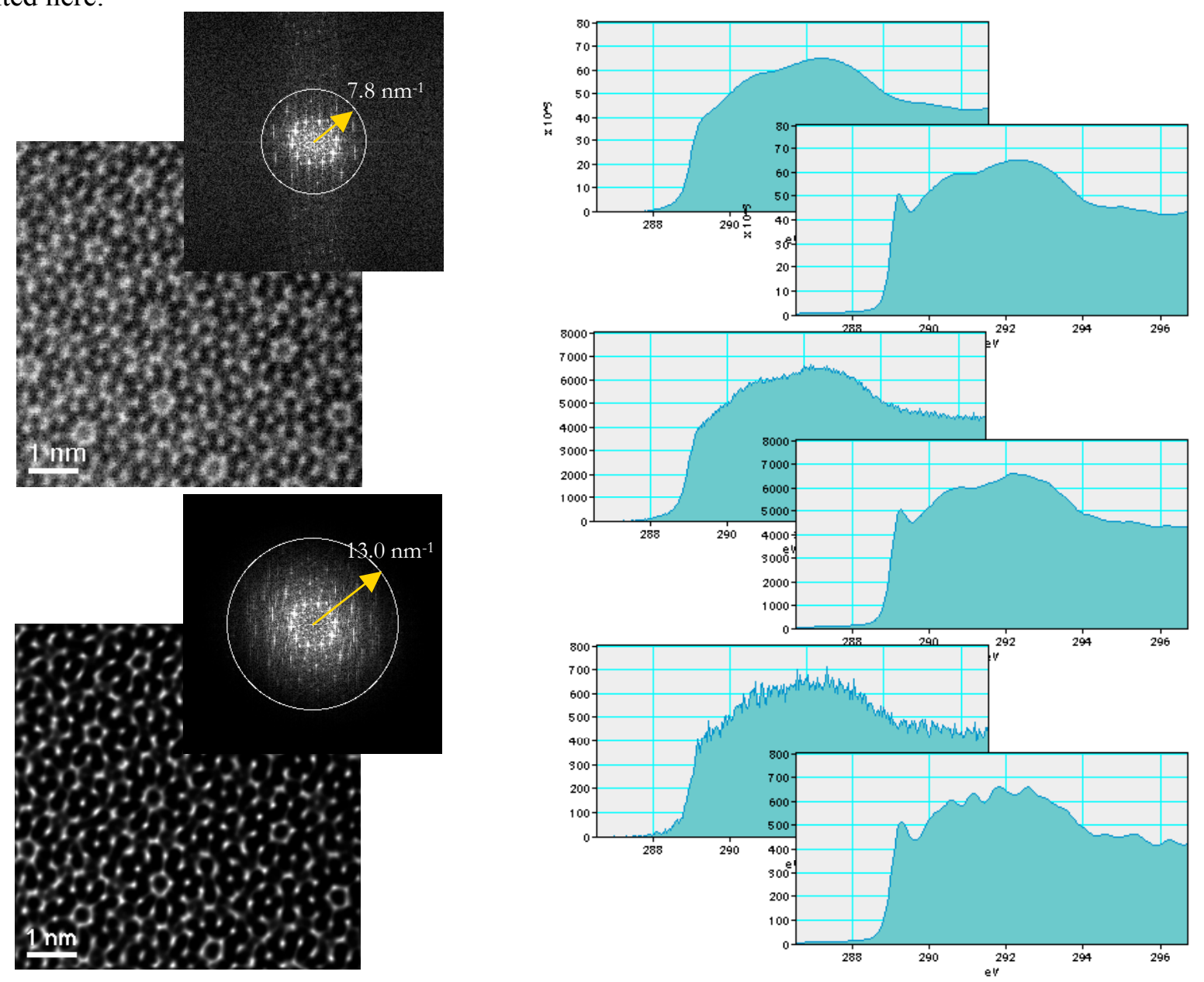

(Left) Fig. 1. Original quasi-crystal image (top) and deconvoluted MEM image (bottom) with a calculated probe assuming $\mathrm{z}=-45 \mathrm{~nm}$ (at 64 cycles). Here, the resolution of the original image is about $7.8 \mathrm{~nm}^{-1}$, while the resolution of the deconvoluted image is about $13.0 \mathrm{~nm}^{-1}$. The original image was obtained by E. Abe using JEOL 2010F (200 kV, Cs: $0.5 \mathrm{~mm}$, probe forming angle: $\sim 12 \mathrm{mrad}$, ADF detector: $50-\sim 110 \mathrm{mrad}$ ).

(Right) Fig. 2. Deconvolution of an original spectrum of carbon K-edge of a diamond (top) and two simulated spectrums artificially reducing counts by ten (middle) and by hundred (bottom). The deconvoluted spectrum from the original spectrum clearly shows an exciton peak. The reduced-by-ten spectrum gives an almost same deconvoluted spectrum. The original spectrum was obtained by Dr. Batson using VG HB501 STEM with a custom designed highresolution spectrometer. 